Bull. Korean Math. Soc. 51 (2014), No. 2, pp. 567-577

http://dx.doi.org/10.4134/BKMS.2014.51.2.567

\title{
LINEAR WEINGARTEN HYPERSURFACES IN RIEMANNIAN SPACE FORMS
}

\author{
Xiaoli Chao and Peijun Wang
}

\begin{abstract}
In this note, we generalize the weak maximum principle in [4] to the case of complete linear Weingarten hypersurface in Riemannian space form $\mathbb{M}^{n+1}(c)(c=1,0,-1)$, and apply it to estimate the norm of the total umbilicity tensor. Furthermore, we will study the linear Weingarten hypersurface in $\mathbb{S}^{n+1}(1)$ with the aid of this weak maximum principle and extend the rigidity results in Li, Suh, Wei [13] and Shu [15] to the case of complete hypersurface.
\end{abstract}

\section{Introduction}

It is well known that many rigidity results have been obtained for hypersurfaces in spheres $([1,6,10,17])$ and in space form $([2,3,12])$ with constant scalar curvature or with constant mean curvature. As a natural generalization of hypersurface with constant scalar curvature or with constant mean curvature, linear Weingarten hypersurface has been studied in many places $([5,8,9,11,13,15,16])$. Recall that a hypersurface in a Riemannian space form is said to be linear Weingarten if its normalized scalar curvature $R$ and mean curvature $H$ satisfy $R=a H+b$ for some constants $a, b \in \mathbb{R}$. In [13], Li, Suh and Wei proved the first rigidity result for linear Weingarten hypersurface in $\mathbb{S}^{n+1}(1)$ under the assumption that the hypersurface is compact. Later, Shu [15] extended this rigidity result to the case of complete linear Weingarten hypersurface with two distinct principle curvatures in real space forms. Recently, Aquino, Lima and Velasquez [5] established a new characterization theorem concerning complete linear Weingarten hypersurface immersed in real space forms under the assumption that the mean curvature attains its maximum along hypersurface and an appropriated restriction on the norm of the traceless part of the second fundamental form.

In this paper, we firstly obtain a weak maximum principle (see Lemma 2.9 below) for the operator $L$ defined by (2.4) which can be regarded as a

Received January 8, 2013; Revised July 13, 2013.

2010 Mathematics Subject Classification. 53C40, 53C42.

Key words and phrases. linear Weingarten hypersurface, maximum principle, space form, Clifford torus, circular cylinder, hyperbolic cylinder. 
generalization of Corollary 10 in [4]. Then, using this maximum principle, we can extend the rigidity results in [13] and [15] to the case of complete linear Weingarten hypersurface without the assumption of two distinct principle curvatures in $\mathbb{M}^{n+1}(c)$. More precisely, we have the following results.

Theorem 1.1. Let $\Sigma^{n}$ be a complete, connected, orientable hypersurface isometrically immersed into a unit sphere $\mathbb{S}^{n+1}(1)$ with $R=a H+b$, where $a, b$ are constants, $a \leq 0$ and $b \geq 1$. Assume further that $0<\sup _{\Sigma}|\Phi|^{2}<+\infty$ and the mean curvature $H$ does not change sign if $b=1$. If $|A|^{2} \leq 2 \sqrt{n-1}$, then $|A|^{2}=2 \sqrt{n-1}$, and $\Sigma^{n}$ is a Clifford torus $\mathbb{S}^{1}\left(\sqrt{1-r^{2}}\right) \times \mathbb{S}^{n-1}(r) \subset \mathbb{S}^{n+1}$.

Moreover, if $\Sigma^{n}$ is $L$-elliptic, we can apply this weak maximum principle to estimate the norm of the total umbilicity tensor.

Theorem 1.2. Let $\Sigma^{n}(n \geq 3)$ be a complete, connected, orientable hypersurface isometrically immersed into a Riemannian space form $\mathbb{M}^{n+1}(c)$ with $R=a H+b$, where $a, b$ are constants and $b>\max \{c, 0\}$. Then either

(I) $\sup _{\Sigma}|\Phi|^{2}=0$ and $\Sigma^{n}$ is a totally umbilical hypersurface, or

(II) $\sup _{\Sigma}|\Phi|^{2} \geq \alpha_{n, c}(a, b)>0$, where $\alpha_{n, c}(a, b)$ is a positive constant depending on $n, c, a, b$. Moreover, the equality $\sup _{\Sigma}|\Phi|^{2}=\alpha_{n, c}(a, b)$ holds and this supremum is attained at some points of $\Sigma^{n}$ if and only if

(1) $\Sigma^{n}$ is a Clifford torus $\mathbb{S}^{1}\left(\sqrt{1-r^{2}}\right) \times \mathbb{S}^{n-1}(r) \subset \mathbb{S}^{n+1}$ when $c=1$;

(2) $\Sigma^{n}$ is circular cylinder $\mathbb{R} \times \mathbb{S}^{n-1}(r) \subset \mathbb{R}^{n+1}$ or $\mathbb{R}^{n-1} \times \mathbb{S}^{1}(r) \subset \mathbb{R}^{n+1}$ when $c=0$;

(3) $\Sigma^{n}$ is hyperbolic cylinder $\mathbb{H}^{n-1}\left(-\sqrt{1+r^{2}}\right) \times \mathbb{S}^{1}(r) \subset \mathbb{H}^{n+1}$ or $\mathbb{H}^{1}\left(-\sqrt{1+r^{2}}\right) \times \mathbb{S}^{n-1}(r) \subset \mathbb{H}^{n+1}$ when $c=-1$.

We also study the case that $\Sigma^{n}$ is $L$-parabolic and obtain a similar theorem as Theorem 1.2 without assuming that the $\operatorname{supremum}_{\sup _{\Sigma}}|\Phi|^{2}=\alpha_{n, c}(a, b)$ is attained on $\Sigma^{n}$.

Theorem 1.3. Let $\Sigma^{n}(n \geq 3)$ be a complete, connected, orientable hypersurface isometrically immersed into a Riemannian space form $\mathbb{M}^{n+1}(c)$ with $R=a H+b$, where $a, b$ are constants, $a \leq 0, b \geq c$ and $b>0$. Assume further that the mean curvature $H$ does not change sign if $b=c$. If $\Sigma^{n}$ is not totally umbilical, then

$$
\sup _{\Sigma^{n}}|\Phi|^{2} \geq \alpha_{n, c}(a, b)>0,
$$

where $\alpha_{n, c}(a, b)$ is the same positive constant as in Theorem 1.2. The equality holds if and only if

(1) $\Sigma^{n}$ is a Clifford torus $\mathbb{S}^{1}\left(\sqrt{1-r^{2}}\right) \times \mathbb{S}^{n-1}(r) \subset \mathbb{S}^{n+1}$ when $c=1$;

(2) $\Sigma^{n}$ is circular cylinder $\mathbb{R} \times \mathbb{S}^{n-1}(r) \subset \mathbb{R}^{n+1}$ or $\mathbb{R}^{n-1} \times \mathbb{S}^{1}(r) \subset \mathbb{R}^{n+1}$ when $c=0$;

(3) $\Sigma^{n}$ is hyperbolic cylinder $\mathbb{H}^{n-1}\left(-\sqrt{1+r^{2}}\right) \times \mathbb{S}^{1}(r) \subset \mathbb{H}^{n+1}$ or $\mathbb{H}^{1}\left(-\sqrt{1+r^{2}}\right) \times \mathbb{S}^{n-1}(r) \subset \mathbb{H}^{n+1}$ when $c=-1$. 
Remark 1.4. When $a=0$, i.e., $\Sigma^{n}$ is a complete hypersurface with constant scalar curvature, Theorem 1.2 and Theorem 1.3 reduce to Theorems 1, 2 and Theorem 3 in [4], respectively.

\section{Preliminaries}

Let $\Sigma^{n}$ be a complete, connected, orientable hypersurface isometrically immersed into a Riemannian space form $\mathbb{M}^{n+1}(c)$ of constant sectional curvature $c=1,0,-1$, and dimensional $n+1$. From [4], we obtain that the Gauss equation of $\Sigma^{n}$ is

$$
n(n-1) R=n(n-1) c+n^{2} H^{2}-|A|^{2},
$$

where $R=a H+b, a, b$ are constants.

Denote by $\Phi$ the totally umbilical tensor of $\Sigma^{n}$, which is given by $\Phi=A-H I$, where $I$ is the identity operator on $T \Sigma^{n}$. Then, by an easy computation, we have $\operatorname{tr}(\Phi)=0$ and

$$
|\Phi|^{2}=|A|^{2}-n H^{2} \geq 0
$$

where the equality $|\Phi|^{2}=0$ holds if and only if $\Sigma^{n}$ is totally umbilical. Combining (2.1) and (2.2), we have

$$
|\Phi|^{2}=n(n-1)\left(H^{2}-a H\right)+n(n-1)(c-b) .
$$

Let $P: T \Sigma^{n} \rightarrow T \Sigma^{n}$ be the operator given by $P=\left(n H-\frac{n-1}{2} a\right) I-A$. It is not difficult to prove that $P$ is self-adjoint. Now we define a operator $L$ associated with $P$ acting on any function $f \in \mathcal{C}^{2}\left(\Sigma^{n}\right)$ by

$$
L(f)=\operatorname{div}(P(\nabla f))=\left(\square-\frac{n-1}{2} a \Delta\right) f,
$$

where $\square$ is the Cheng-Yau's operator in [10], i.e.,

$$
\square f=\sum\left(n H \delta_{i j}-h_{i j}\right) f_{i j} .
$$

Thus, $L$ is a second-order differential operator and $L$ is elliptic or parabolic if and only if $P$ is positive definite or non-negative definite.

Lemma 2.1. Let $\Sigma^{n} \hookrightarrow \mathbb{M}^{n+1}(c)$ be an oriented isometrically immersed hypersurface with $R=a H+b$, where $a, b$ are constants. Then

$$
L(n H)=|\nabla A|^{2}-n^{2}|\nabla H|^{2}+n H \operatorname{tr}\left(A^{3}\right)-|A|^{4}+n c\left(|A|^{2}-n H^{2}\right) .
$$

Proof. From the Corollary 3.3 in [7], we have

$$
\begin{aligned}
& \square(n H) \\
= & \frac{1}{2} \Delta n(n-1) R+|\nabla A|^{2}-n^{2}|\nabla H|^{2}+n H \operatorname{tr}\left(A^{3}\right)-|A|^{4}+n c\left(|A|^{2}-n H^{2}\right) \\
= & \frac{n(n-1) a}{2} \Delta H+|\nabla A|^{2}-n^{2}|\nabla H|^{2}+n H \operatorname{tr}\left(A^{3}\right)-|A|^{4}+n c\left(|A|^{2}-n H^{2}\right) .
\end{aligned}
$$


Then

$$
\begin{aligned}
L(n H) & =\square(n H)-\frac{n-1}{2} a \Delta(n H)=\square(n H)-\frac{n(n-1)}{2} a \Delta(H) \\
& =|\nabla A|^{2}-n^{2}|\nabla H|^{2}+n H \operatorname{tr}\left(A^{3}\right)-|A|^{4}+n c\left(|A|^{2}-n H^{2}\right) .
\end{aligned}
$$

Lemma 2.2. Let $\Sigma^{n} \hookrightarrow \mathbb{M}^{n+1}(c)$ be an oriented isometrically immersed hypersurface with $R=a H+b$, where $a, b$ are constants and $b>c$. Then $L$ is elliptic.

Proof. From (2.1), we have

$$
|A|^{2}=n^{2} H^{2}+n(n-1)(c-a H-b),
$$

and then

$$
n H[n H-(n-1) a]=n(n-1)(b-c)+|A|^{2}>0,
$$

if $b>c$. Thus the connectedness of $\Sigma^{n}$ implies that $H$ doesn't change sign if $b>c$. So we can choose the orientation of $\Sigma^{n}$ such that $H>0$ on $\Sigma^{n}$. Let $\mu_{i}$ be the eigenvalue of $P$ at every point $p \in \Sigma^{n}$. Then $\mu_{i}=n H-\frac{n-1}{2} a-\lambda_{i}(i=$ $1,2, \ldots, n)$, where $\lambda_{i}(i=1,2, \ldots, n)$ are the principle curvatures of $\Sigma^{n}$. Since $H \neq 0$, we can obtain from (2.1) that

$$
-\frac{n-1}{2} a=\frac{1}{2 n H}\left(|A|^{2}-n^{2} H^{2}+n(n-1)(b-c)\right) .
$$

Therefore, for every $i$,

$$
\begin{aligned}
\mu_{i} & =n H-\frac{n-1}{2} a-\lambda_{i} \\
& =n H-\lambda_{i}+\frac{1}{2 n H}\left(|A|^{2}-n^{2} H^{2}+n(n-1)(b-c)\right) \\
& =\frac{n-1}{2 H}(b-c)+\frac{1}{2 n H}\left(|A|^{2}+n^{2} H^{2}-2 n H \lambda_{i}\right)
\end{aligned}
$$

Observe now that

$$
\begin{aligned}
|A|^{2}+n^{2} H^{2}-2 n H \lambda_{i} & =\sum_{j=1}^{n} \lambda_{j}^{2}+\left(\sum_{j=1}^{n} \lambda_{j}\right)^{2}-2\left(\sum_{j=1}^{n} \lambda_{j}\right) \lambda_{i} \\
& =\sum_{j=1, j \neq i}^{n} \lambda_{j}^{2}+\left(\sum_{j=1, j \neq i}^{n} \lambda_{j}\right)^{2} \geq 0 .
\end{aligned}
$$

So (2.7), (2.8) and $b>c$ imply that $\mu_{i}>0$ for each $i$ and $L$ is elliptic.

Remark 2.3. If we assume further that $H$ doesn't change sign if $b=c$, then from the proof of Lemma 2.2 , we can easily obtain that $\mu_{i} \geq 0$ if $b \geq c$, i.e., $L$ is parabolic if $b \geq c$. 
Lemma $2.4([14])$. Let $\beta_{1}, \beta_{2}, \ldots, \beta_{n}$ be real numbers such that $\sum_{i=1}^{n} \beta_{i}=0$. Then

$$
-\frac{n-2}{\sqrt{n(n-1)}}\left(\sum_{i=1}^{n} \beta_{i}^{2}\right)^{3 / 2} \leq \sum_{i=1}^{n} \beta_{i}^{3} \leq \frac{n-2}{\sqrt{n(n-1)}}\left(\sum_{i=1}^{n} \beta_{i}^{2}\right)^{3 / 2} .
$$

Moreover, equality holds if and only if at least $(n-1)$ of the $\beta_{i}^{\prime} s$ are equal.

Lemma 2.5. Let $\Sigma^{n} \hookrightarrow \mathbb{M}^{n+1}(c)$ be an oriented isometrically immersed hypersurface with $R=a H+b$, where $a, b$ are constants and $(n-1) a^{2}+4 n(b-c) \geq 0$. Then

$$
|\nabla A|^{2} \geq n^{2}|\nabla H|^{2}
$$

Proof. Taking the covariant derivative on the both sides of (2.6), we obtain

$$
2 \sum_{i, j} h_{i j} h_{i j k}=2 n^{2} H H_{k}-n(n-1) a H_{k} .
$$

Then

$$
4|A|^{2}|\nabla A|^{2} \geq 4 \sum_{k}\left(\sum_{i, j} h_{i j} h_{i j k}\right)^{2}=\left[2 n^{2} H-n(n-1) a\right]^{2}|\nabla H|^{2} .
$$

On the other hand, we have

$$
\begin{aligned}
& {\left[2 n^{2} H-n(n-1) a\right]^{2}-4 n^{2}|A|^{2} } \\
= & 4 n^{4} H^{2}-4 n^{3}(n-1) a H+n^{2}(n-1)^{2} a^{2}-4 n^{2}\left[n^{2} H^{2}+n(n-1)(c-a H-b)\right] \\
= & n^{2}(n-1)^{2} a^{2}+4 n^{3}(n-1)(b-c) \geq 0 .
\end{aligned}
$$

Combining (2.11) and (2.12), we obtain

$$
|\nabla A|^{2} \geq n^{2}|\nabla H|^{2}
$$

Lemma 2.6. Let $\Sigma^{n} \hookrightarrow \mathbb{M}^{n+1}(c)$ be an oriented isometrically immersed hypersurface with $R=a H+b$, where $a, b$ are constants and $b \geq c$. Then

$$
\frac{1}{2} L\left(|\Phi|^{2}\right) \geq(n-1)|\Phi|^{2} \varphi_{a, b}(|\Phi|) \sqrt{\frac{|\Phi|^{2}}{n(n-1)}+\left(b+\frac{a^{2}}{4}-c\right)},
$$

where

$$
\begin{aligned}
& \varphi_{a, b}(x) \\
= & -\frac{n-2}{n-1} x^{2}+\left(-\frac{n-2}{n-1} x+\frac{n a}{\sqrt{n(n-1)}}\right) \sqrt{x^{2}+n(n-1)\left(b+\frac{a^{2}}{4}-c\right)} \\
& -\frac{n(n-2)}{\sqrt{n(n-1)}} \frac{a}{2} x+n\left(b+\frac{a^{2}}{2}\right), \quad x \geq 0 .
\end{aligned}
$$


Proof. From (2.3), (2.5) and the fact that $P$ is non-negative definite, we have

$$
\begin{aligned}
& \frac{n}{2(n-1)} L\left(|\Phi|^{2}\right) \\
= & \frac{1}{2} L\left(n^{2} H^{2}-n^{2} a H\right) \\
= & n H L(n H)+n^{2}\langle P(\nabla H), \nabla H\rangle-\frac{n a}{2} L(n H) \\
\geq & \left(n H-\frac{n a}{2}\right) L(n H) \\
= & \left(n H-\frac{n a}{2}\right)\left[|\nabla A|^{2}-n^{2}|\nabla H|^{2}+n H \operatorname{tr}\left(A^{3}\right)-|A|^{4}+n c\left(|A|^{2}-n H^{2}\right)\right] .
\end{aligned}
$$

Since $H>0$ as shown in Lemma 2.2, we conclude from (2.10) and (2.15) that

(2.16) $\frac{1}{2(n-1)} L\left(|\Phi|^{2}\right) \geq\left(H-\frac{a}{2}\right)\left[n H \operatorname{tr}\left(A^{3}\right)-|A|^{4}+n c\left(|A|^{2}-n H^{2}\right)\right]$.

By a direct computation, we can easily obtain

$$
\operatorname{tr}\left(A^{3}\right)=\operatorname{tr}\left(\Phi^{3}\right)+3 H|\Phi|^{2}+n H^{3}
$$

and

$$
|A|^{4}=|\Phi|^{4}+2 n H^{2}|\Phi|^{2}+n^{2} H^{4} .
$$

Substituting (2.17) and (2.18) into (2.16), we have

$$
\frac{1}{2(n-1)} L\left(|\Phi|^{2}\right) \geq\left(H-\frac{a}{2}\right)\left[n H t r\left(\Phi^{3}\right)-|\Phi|^{4}+n\left(c+H^{2}\right)|\Phi|^{2}\right] .
$$

Since $\operatorname{tr}(\Phi)=0$, then from Lemma 2.4, we obtain

$$
\left|\operatorname{tr}\left(\Phi^{3}\right)\right| \leq \frac{n-2}{\sqrt{n(n-1)}}|\Phi|^{3},
$$

and

$$
n H \operatorname{tr}\left(\Phi^{3}\right) \geq-n H\left|\operatorname{tr}\left(\Phi^{3}\right)\right| \geq-\frac{n(n-2)}{\sqrt{n(n-1)}} H|\Phi|^{3} .
$$

Combining (2.21) and (2.19), we obtain

$$
\frac{1}{2(n-1)} L\left(|\Phi|^{2}\right) \geq\left(H-\frac{a}{2}\right)|\Phi|^{2}\left[-|\Phi|^{2}-\frac{n(n-2)}{\sqrt{n(n-1)}} H|\Phi|+n\left(c+H^{2}\right)\right] .
$$

From (2.3) and by direct computation, we have

$$
H=\frac{a}{2}+\sqrt{\frac{|\Phi|^{2}}{n(n-1)}+\left(b+\frac{a^{2}}{4}-c\right)} .
$$


Substituting (2.23) into (2.22), we obtain

$$
\frac{1}{2(n-1)} L\left(|\Phi|^{2}\right) \geq|\Phi|^{2} \varphi_{a, b}(|\Phi|) \sqrt{\frac{|\Phi|^{2}}{n(n-1)}+\left(b+\frac{a^{2}}{4}-c\right)},
$$

which is just (2.13).

Remark 2.7. By an easy computation, we can prove that $\varphi_{a, b}(x)$ is a decreasing function of $x$ for $x \geq 0$.

Lemma $2.8([4])$. Let $\left(\Sigma^{n},\langle\rangle,\right)$ be a complete Riemannian manifold, let o be a reference point in $\Sigma^{n}$, and let $r(p)$ be the distance function from o. Let $h$ be a symmetric $(0,2)$ tensor field on $\Sigma^{n}$ and set $\sharp: T^{*} \Sigma^{n} \rightarrow T \Sigma^{n}$ to denote the musical isomorphism, so that $h(X, \cdot)^{\#}$ is the vector field on $\Sigma^{n}$ defined by

$$
\left\langle h(X, \cdot)^{\sharp}, Y\right\rangle=h(X, Y)
$$

for every $Y \in T_{p} \Sigma^{n}$ and $p \in \Sigma^{n}$. Assume that, for some positive continuous function $h_{+}$defined on $[0 .+\infty)$, the tensor $h$ satisfies

$$
0 \leq h(X, X) \leq h_{+}(r)
$$

for every $X \in T_{p} \Sigma^{n}$ with $|X|=1$ and every $p \in \partial B_{r}$, where $\partial B_{r}$ denotes the geodesic ball of radius $r$ centered at $o$. Set

$$
h_{+}^{*}(r)=\sup _{s \leq r} h_{+}(s) .
$$

Given $f \in \mathcal{C}^{0}(\mathbb{R})$, assume that $\mu \in \mathcal{C}^{2}\left(\Sigma^{n}\right)$ satisfies $\mu^{*}=\sup _{\Sigma^{n}} \mu<+\infty$ and

$$
\mathcal{L}(\mu)=\operatorname{div}\left(h(\nabla \mu, \cdot)^{\sharp}\right) \geq f(\mu)
$$

on the set $\Omega_{\gamma}=\left\{p \in \Sigma^{n}: \mu(p)>\gamma\right\}$ for some $\gamma<\mu^{*}$. If

$$
\lim _{r \rightarrow+\infty} \frac{h_{+}^{*}(r)}{r^{2}}=0
$$

and

$$
\liminf _{r \rightarrow+\infty} \frac{h_{+}^{*}(r) \log \operatorname{vol} B_{r}}{r^{2}}<+\infty
$$

then $f\left(\mu^{*}\right) \leq 0$

By applying Lemma 2.8 to the operator $L$, we shall obtain the following weak maximum principle for $L$.

Lemma 2.9. Let $\Sigma^{n} \hookrightarrow \mathbb{M}^{n+1}(c)$ be an oriented isometrically immersed hypersurface with $R=a H+b$, where $a, b$ are constants. Assume that $\sup _{\Sigma^{n}}|\Phi|^{2}<$ $+\infty$. If $\mu \in \mathcal{C}^{2}\left(\Sigma^{n}\right)$ satisfies $\mu^{*}=\sup _{\Sigma^{n}} \mu<+\infty$ and for a given $f \in \mathcal{C}^{0}(\mathbb{R})$,

$$
L(\mu) \geq f(\mu)
$$

on the set $\Omega_{\gamma}=\left\{p \in \Sigma^{n}: \mu(p)>\gamma\right\}$ for some $\gamma<\mu^{*}$, then $f\left(\mu^{*}\right) \leq 0$. 
Proof. Set

$$
h(X, Y)=\langle P X, Y\rangle
$$

Then

$$
h(\nabla \mu, \cdot)^{\sharp}=P(\nabla \mu)
$$

for arbitrary $\mu \in \mathcal{C}^{2}\left(\Sigma^{n}\right)$. Therefore, $\mathcal{L}(\mu)=\operatorname{div}\left(h(\nabla \mu, \cdot)^{\sharp}\right)=L(\mu)$. Since $h_{+}(r)=2 n \sup _{\partial B_{r}} H-\frac{n-1}{2} a$, then

$$
h_{+}^{*}(r)=2 n \sup _{B_{r}} H-\frac{n-1}{2} a .
$$

If $\sup _{\Sigma^{n}}|\Phi|^{2}<+\infty$, then from $(2.3)$, we have $\sup _{\Sigma^{n}} H<+\infty$. Thus

$$
h_{+}^{*}(r)=2 n \sup _{B_{r}} H-\frac{n-1}{2} a \leq 2 n \sup _{\Sigma^{n}} H-\frac{n-1}{2} a .
$$

So $(2.26)$ is satisfied.

On the other hand, if $\sup _{\Sigma^{n}}|\Phi|^{2}<+\infty$, then $\lambda_{i}^{2} \leq|A|^{2}<+\infty$, we have

$$
\begin{aligned}
\left|\operatorname{Ric}\left(e_{i}\right)\right| & =\left|\sum_{i \neq j} R_{i j i j}\right|=\left|\sum_{i \neq j}\left(c+\lambda_{i} \lambda_{j}\right)\right| \leq n(n-1) c+\sum_{i \neq j}\left|\lambda_{i} \lambda_{j}\right| \\
& \leq n(n-1) c+\sum_{i \neq j} \sup _{\Sigma^{n}}\left|\lambda_{i}\right| \sup _{\Sigma^{n}}\left|\lambda_{j}\right|<+\infty .
\end{aligned}
$$

Thus the Ricci curvature of $\Sigma^{n}$ is bounded from above. Note that $\Sigma^{n}$ is complete. Then by Bishop's volume comparison theorem, we have

$$
\liminf _{r \rightarrow+\infty} \frac{\log \operatorname{vol} B_{r}}{r^{2}}<+\infty .
$$

Combining (2.29) and (2.28), we show (2.27) holds. By Lemma 2.8, we have $f\left(\mu^{*}\right) \leq 0$.

\section{Proof of the main theorems}

Proof of Theorem 1.1. For any positive number $d$, we have

$$
|H||\Phi| \leq \frac{d}{2} H^{2}+\frac{1}{2 d}|\Phi|^{2} .
$$

Combining (2.2), (2.22) and (3.1) with $d=\frac{n+2 \sqrt{n-1}}{n-2} \sqrt{n}$, we obtain

$$
\begin{aligned}
& \frac{1}{2(n-1)} L\left(|\Phi|^{2}\right) \\
\geq & \left(H-\frac{a}{2}\right)|\Phi|^{2}\left[-|\Phi|^{2}-\frac{n(n-2) d}{2 \sqrt{n(n-1)}} H^{2}-\frac{n(n-2)}{2 d \sqrt{n(n-1)}}|\Phi|^{2}+n\left(1+H^{2}\right)\right] \\
= & \left(H-\frac{a}{2}\right)|\Phi|^{2}\left\{\left[n-\left(1+\frac{n(n-2)}{2 d \sqrt{n(n-1)}}\right)|A|^{2}\right]\right.
\end{aligned}
$$




$$
\begin{aligned}
& \left.+\left(2-\frac{(n-2) d}{2 \sqrt{n(n-1)}}+\frac{n-2}{2 d \sqrt{n(n-1)}}\right) n H^{2}\right\} \\
= & \left(H-\frac{a}{2}\right)|\Phi|^{2}\left[n-\frac{n}{2 \sqrt{n-1}}|A|^{2}\right],
\end{aligned}
$$

then

$$
L(\mu) \geq 2(n-1)\left(H-\frac{a}{2}\right) \mu\left[n-\frac{n}{2 \sqrt{n-1}}|A|^{2}\right]=f(\mu),
$$

where $\mu=|\Phi|^{2}$. Set $\mu^{*}=\sup _{\Sigma^{n}} \mu=\sup _{\Sigma^{n}}|\Phi|^{2}$. Then applying Lemma 2.9 to the operator $L$, we have

$$
f\left(\mu^{*}\right)=2(n-1)\left(H-\frac{a}{2}\right) \mu^{*}\left[n-\frac{n}{2 \sqrt{n-1}}|A|^{2}\right] \leq 0 .
$$

Since $H>0$ as shown in Lemma $2.2, a \leq 0$ and $\mu^{*}=\sup _{\Sigma^{n}}|\Phi|^{2}>0$, we obtain from (3.4) that

$$
|A|^{2} \geq 2 \sqrt{n-1}
$$

Noticing that $|A|^{2} \leq 2 \sqrt{n-1}$ as the assumption shows, we have

$$
|A|^{2}=2 \sqrt{n-1} \text {. }
$$

Then it follows from (2.6) and Lemma 2.4 that $\Sigma^{n}$ has two distinct constant principle curvatures and one of which is simple. By the classical result in [4], we obtain that $\Sigma^{n}$ is a Clifford torus $\mathbb{S}^{1}\left(\sqrt{1-r^{2}}\right) \times \mathbb{S}^{n-1}(r) \subset \mathbb{S}^{n+1}$.

Proof of Theorem 1.2. (1) If $\sup _{\Sigma^{n}}|\Phi|^{2}=0$, i.e., $|A|^{2}=n H^{2}$, then $\Sigma^{n}$ is a totally umbilical hypersurface, and thus (I) holds;

(2) If $\sup _{\Sigma^{n}}|\Phi|^{2}=+\infty$, then (II) holds trivially;

(3) If $0<\sup _{\Sigma^{n}}|\Phi|^{2}<+\infty$, let $\mu=|\Phi|^{2}$. Lemma 2.6 tells us that

$$
L(\mu) \geq 2(n-1) \mu \varphi_{a, b}(\sqrt{\mu}) \sqrt{\frac{\mu}{n(n-1)}+\left(b+\frac{a^{2}}{4}-c\right)}=f(\mu),
$$

where $\varphi_{a, b}(x)$ is defined by (2.14). Then from Lemma 2.9, we get

$$
f\left(\mu^{*}\right)=2(n-1) \mu^{*} \varphi_{a, b}\left(\sqrt{\mu^{*}}\right) \sqrt{\frac{\mu^{*}}{n(n-1)}+\left(b+\frac{a^{2}}{4}-c\right)} \leq 0 .
$$

Since $\mu^{*}=\sup _{\Sigma^{n}} \mu=\sup _{\Sigma^{n}}|\Phi|^{2}>0$ and $b>c$, then from (3.8), we deduce that

$$
\varphi_{a, b}\left(\sqrt{\mu^{*}}\right) \leq 0 .
$$

Since $b>0$, we have $\varphi_{a, b}(0)>0$. Also note that $\varphi_{a, b}(x)$ is strictly decreasing for $x \geq 0$ and $\varphi_{a, b}(x)<0$ if $x$ is sufficiently large. By the continuity of $\varphi_{a, b}(x)$, we may assume the equation $\varphi_{a, b}(x)=0$ attains its positive root at $\sqrt{\alpha_{n, c}(a, b)}$, 
where $\alpha_{n, c}(a, b)$ is a positive constant depending on $n, c, a, b$. Then from (3.9), we must have

$$
\sup _{\Sigma^{n}}|\Phi|^{2}=\mu^{*} \geq \alpha_{n, c}(a, b) .
$$

Thus we complete the proof of the inequality in (II) of Theorem 1.2.

Moreover, the equality $\sup _{\Sigma^{n}}|\Phi|^{2}=\alpha_{n, c}(a, b)>0$ holds if and only if $\varphi_{a, b}\left(\sqrt{\mu^{*}}\right)=0$, then $\varphi_{a, b}(\sqrt{\mu}) \geq 0$ on $\Sigma^{n}$. Therefore, from (3.7), we have that $L(\mu) \geq 0$ on $\Sigma^{n}$. Also, from Lemma 2.2 , we know $L$ is elliptic. As a result, if this supremum is attained at some point of $\Sigma^{n}$, then by the maximum principle, we know $\mu=|\Phi|^{2}$ must be a constant, i.e., $|\Phi|^{2} \equiv \alpha_{n, c}(a, b)$. Since $L$ is a second-order differential operator, then $L\left(|\Phi|^{2}\right)=0$. Therefore all the inequalities in the proof of Lemma 2.6 become equalities. Then from (2.15) and (2.6), we obtain that $H$ and $|A|^{2}$ are constants. In particular, from equality (2.20) and Lemma 2.4, we know that $\Sigma^{n}$ has two distinct constant principle curvatures and one of which is simple. Thus $\Sigma^{n}$ is an isoparametric hypersurface. Then, by the classical result in [4], we deduce that $\Sigma^{n}$ satisfies the three following standard product embeddings:

(1) $\Sigma^{n}$ be a Clifford torus $\mathbb{S}^{1}\left(\sqrt{1-r^{2}}\right) \times \mathbb{S}^{n-1}(r) \subset \mathbb{S}^{n+1}$ if $c=1$;

(2) $\Sigma^{n}$ be circular cylinder $\mathbb{R} \times \mathbb{S}^{n-1}(r) \subset \mathbb{R}^{n+1}$ or $\mathbb{R}^{n-1} \times \mathbb{S}^{1}(r) \subset \mathbb{R}^{n+1}$ if $c=0$

(3) $\Sigma^{n}$ be hyperbolic cylinder $\mathbb{H}^{n-1}\left(-\sqrt{1+r^{2}}\right) \times \mathbb{S}^{1}(r) \subset \mathbb{H}^{n+1}$ or $\mathbb{H}^{1}\left(-\sqrt{1+r^{2}}\right) \times \mathbb{S}^{n-1}(r) \subset \mathbb{H}^{n+1}$ if $c=-1$.

Proof of Theorem 1.3. First, Theorem 1.3 holds trivially if $\sup _{\Sigma^{n}}|\Phi|^{2}=+\infty$. So we just need to consider the case of $0<\sup _{\Sigma^{n}}|\Phi|^{2}<+\infty$. From the first part of the proof of Theorem 1.2, we easily obtain that $\sup _{\Sigma^{n}}|\Phi|^{2} \geq \alpha_{n, c}(a, b)$. Then from $\Sigma^{n}$ is $L$-parabolic, we conclude that $|\Phi|^{2}$ must be constants and equal to $\alpha_{n, c}(a, b)$. The rest of proof can follow as the proof of Theorem 1.2.

Acknowledgements. The authors would like to thank the referee for giving some valuable suggestions which improve the paper.

\section{References}

[1] H. Alencar and M. do Carmo, Hypersurfaces with constant mean curvature in spheres, Proc. Amer. Math. Soc. 120 (1994), no. 4, 1223-1229.

[2] L. J. Alias and S. C. Garcia-Martinez, On the scalar curvature of constant mean curvature hypersurfaces in space forms, J. Math. Anal. Appl. 363 (2010), no. 2, 579-587.

[3] _ An estimate for the scalar curvature of constant mean curvature hypersurfaces in space forms, Geom. Dedicata 156 (2012), 31-47.

[4] L. J. Alias, S. C. Garcia-Martinez, and M. Rigoli, A maximum principle for hypersurfaces with constant scalar curvature and applications, Ann. Global Anal. Geom. 41 (2012), no. 3, 307-320.

[5] C. Aquino, H. de Lima, and M. Velasquez, A new characterization of complete linear Weingarten hypersurfaces in real space forms, Pacific J. Math. 261 (2013), no. 1, 33-43.

[6] A. Brasil Jr., A. G. Colares, and O. Palmas, Complete hypersurfaces with constant scalar curvature in spheres, Monatsh. Math. 161 (2010), no. 4, 369-380. 
[7] A. Caminha, On hypersurfaces into Riemannian spaces of constant sectional curvature, Kodai Math. J. 29 (2006), no. 2, 185-210.

[8] X. L. Chao, On complete spacelike submanifolds in semi-Riemannian space forms with parallel normalized mean curvature vector, Kodai Math. J. 34 (2011), no. 1, 42-54.

[9] H. Chen and X. F. Wang, Stability and eigenvalue estimates of linear Weingarten hypersurfaces in a sphere, J. Math. Anal. Appl. 397 (2013), no. 2, 658-670.

[10] S. Y. Cheng and S. T. Yau, Hypersurfaces with constant scalar curvature, Math. Ann. 225 (1977), no. 3, 195-204.

[11] A. V. Corro, W. Ferreira, and K. Tenenblat, Ribaucour transformations for constant mean curvature and linear Weingarten surfaces, Pacific J. Math. 212 (2003), no. 2, 265-297.

[12] H. Z. Li, Hypersurfaces with constant scalar curvature in space forms, Math. Ann. 305 (1996), no. 4, 665-672.

[13] H. Z. Li, Y. J. Suh, and G. X. Wei, Linear Weingarten hypersurfaces in a unit sphere, Bull. Korean Math. Soc. 46 (2009), no. 2, 321-329.

[14] M. Okumura, Hypersurfaces and a pinching problem on the second fundamental tensor, Amer. J. Math. 96 (1974), 207-213.

[15] S. C. Shu, Linear Weingarten hypersurfaces in a real space form, Glasg. Math. J. 52 (2010), no. 3, 635-648.

[16] D. Yang, Linear Weingarten spacelike hypersurfaces in locally symmetric Lorentz space, Bull. Korean Math. Soc. 49 (2012), no. 2, 271-284.

[17] Q. Zhang, Scalar curvature of hypersurfaces with constant mean curvature in spheres, Glasg. Math. J. 54 (2012), no. 1, 67-75.

\section{Xiaoli Chao}

Department of MATHEMATiCs

SOUTHEAST UNIVERSiTy

210096 Nanjing, P. R. China

E-mail address: xlchao@seu.edu.cn

PEIJUN WANG

Department of Mathematics

SOUTHEAST UNIVERSITY

210096 Nanjing, P. R. China

E-mail address: pjw4047778@163.com 